\title{
Biometrics and Biosecurity
}

\section{Tai-hoon Kim, ${ }^{1}$ Sabah Mohammed, ${ }^{2}$ Carlos Ramos, ${ }^{3}$ Osvaldo Gervasi, ${ }^{4}$ Wai-Chi Fang, ${ }^{5}$ and Adrian Stoica ${ }^{6}$}

${ }^{1}$ GVSA and University of Tasmania, 20 Virgina Court, Sandy Bay, Tasmania, Australia

${ }^{2}$ Lakehead University, 955 Oliver Road, Thunder Bay, ON, Canada P7B 5E1

${ }^{3}$ Instituto Superior de Engenharia do Porto GECAD, 4200-072 Porto, Portugal

${ }^{4}$ University of Perugia, Via Vanvitelli 1, I-06123 Perugia, Italy

${ }^{5}$ National Chiao Tung University, 1001 Ta Hsueh Road, Hsinchu 300, Taiwan

${ }^{6}$ JPL NASA, The California Institute of Technology, Le Canada Flintridge, CA 91011, USA

Correspondence should be addressed to Tai-hoon Kim, taihoonn@paran.com

Received 9 August 2012; Accepted 9 August 2012

Copyright ( 2012 Tai-hoon Kim et al. This is an open access article distributed under the Creative Commons Attribution License, which permits unrestricted use, distribution, and reproduction in any medium, provided the original work is properly cited.

We are very happy to publish this special issue of a Journal of Biomedicine and Biotechnology published by Hindawi Publishing Corporation.

This special issue contains 23 articles among totally 192 papers accepted in the BSBT 2011, AITS 2012, ISA 2012, and UCMA 2012 postsubmission. Achieving such a high quality of papers would have been impossible without the huge work that was undertaken by the International Program Committee members and external reviewers. We take this opportunity to thank them for their great support and cooperation.

Biometrics and Biosecurity focused on the various aspects of advances in Biometrics and Biosecurity. This special issue will provide a chance for academic and industry professionals to discuss recent progress, problems, and solutions in the area of biometrics and its application, biosecurity measures, biosafety protocols, including development, implementation, strategies, and policies.

In "Anatomy of biometric passports," authors summed up relevant details of the electronic passports implementation in the Czech Republic. This work will be used as a basis for the next steps in an analysis of hardware (microscopic analysis, side channel analysis, etc.) and software (protocols analysis, firmware analysis, etc.) of such passports (in fact of the RFID chips) that will be performed soon.

In "Security analysis and enhancements of an effective biometric-based remote User authentication scheme using smart cards," authors analyzed the security of Das's scheme.
Authors have shown that Das's scheme is not secure against the various attacks and fails to provide mutual authentication between the user and the server. Also, authors proposed the enhanced scheme to overcome these security weaknesses, while preserving all their merits, even if the secret information stored in the smart card is revealed.

Paper "Advanced pulse oximetry system for remote monitoring and management" proposes an advanced pulse oximetry system for remote monitoring and management. The system consists of a networked pulse oximeter and a personal monitoring server. The proposed pulse oximeter measures a patient's pulse oximetry data and transmits the data to the personal monitoring server.

In "Real-time clinical decision support system with data stream mining," a new system is introduced that can analyze medical data streams and can make real-time prediction. This system is based on stream mining algorithm called VFDT. The VFDT is extended with the capability using pointers to allow the decision tree to remember the mapping relationship between leaf nodes and the history records.

The paper "Construction of a smart medication dispenser with high degree of scalability and remote manageability," proposed a smart medication dispenser having a high degree of scalability and remote manageability. Authors constructed the dispenser to have extensible hardware architecture for achieving scalability, and we install an agent program in it for achieving remote manageability. 
The paper "A hybrid technique for medical image segmentation," proposed a hybrid method for magnetic resonance (MR) image segmentation. Authors first removed impulsive noise inherent in MR images by utilizing a vector median filter. Subsequently, Otsu thresholding was used as an initial coarse segmentation method that finds the homogeneous regions of the input image. Finally, an enhanced suppressed fuzzy c-means was used to partition brain MR images into multiple segments, which employed an optimal suppression factor for the perfect clustering in the given data set.

In the paper, "A survey and proposed framework on the soft biometrics technique for human identification in intelligent video surveillance system," the identification technique using biometrics suitable for video surveillance system was analyzed. In addition, the framework was proposed to complement the problems of decreasing recognition performance due to lighting, occlusion, and shadow.

In the paper, "A classification method of normal and overweight females based on facial features for automated medical applications," authors demonstrated that it is possible to predict body mass index (BMI) status using facial characteristics. Authors' research results provided useful information for studies of obesity and facial characteristics and might provide useful clues in the development of applications for alternative diagnosis of obesity in remote healthcare.

The aim of this paper, "A new method of diagnosing constitutional types based on vocal and facial features for personalized medicine" is to develop an accurate constitution diagnostic method based solely on the individual's physical characteristics, irrespective of psychologic traits, characteristics of clinical medicine, and genetic factors. In this paper, authors suggested a novel method for diagnosing constitutional types using only speech and face characteristics.

In "Secure remote health monitoring with unreliable mobile devices," authors designed a framework for secure remote health-monitoring systems. Specifically, authors (i) built a realistic risk model for sensor-data quality by interacting with health professionals, (ii) developed protocols and mechanisms for data protection and quality assurance, and (iii) proposed a new health-monitoring architecture that is secure despite the weaknesses of common personal devices.

The paper, "A collaborative molecular modeling environment using a virtual tunneling service" proposed a collaborative molecular modeling environment to integrate different molecule modeling systems using a virtual tunneling service. Authors integrated Co-Coot, which is a collaborative crystallographic object-oriented toolkit, with VRMMS, which is a virtual reality molecular modeling system, through a collaborative tunneling system.

The paper, "A privacy-preserved analytical method for eHealth database with minimized information loss" proposed the Hiatus Tailor (HT) system. By using the execution chain graph (ECG) to progressively deidentify data, people's privacy can be protected. The name Hiatus Tailor refers to the fact that the proposed system is capable of identifying the missing element within the system and fixing it.

In "An integrated gateway for various PHDs in uhealthcare environments," authors proposed an integrated gateway for various personal health devices (PHDs). The evaluation results showed that the size of separate messages from various PHDs is reduced through the integration process, and the process does not require much time; the integration time is negligible.

The paper "Criminal genomic pragmatism: prisoners' representations of DNA technology and biosecurity," explores the relationship between biometrics (specifically DNA material) and biosecurity (the risks and benefits of DNA technology) in forensic applications from the point of view of convicted offenders.

In the paper, "Bayesian integration of isotope ratio for geographic sourcing of castor beans," authors investigated two sources of isotope ratio (IR) data that may provide insight into the region of origin for castor beans; (i) light element (C, $\mathrm{N}, \mathrm{O}$, and $\mathrm{H}$ ) stable isotope ratios (LeIR) and (ii) Sr isotope ratios (SrIR). Both data types have been used to associate plant and animal material with regions of origin.

In the paper "Influence of skin diseases on fingerprint recognition," authors prepared a new collection of fingerprints with skin diseases from patients, oriented not only on dactyloscopic fingerprints, but also on live fingerprint images from different fingerprint scanners. The resulting images will be used for quality assessment, if the quality will be acceptable for minutiae extraction and comparison based on minutiae.

In the paper, "Finger-vein recognition based on $(2 D)^{2} P C A$ and metric learning," $(2 \mathrm{D})^{2} \mathrm{PCA}$ is applied to extract features of finger veins, based on a new recognition method which is proposed in conjunction with metric learning. It learns a KNN classifier for each individual, which is different from the traditional methods where a fixed threshold is employed for all individuals.

In "Using hierarchical time series clustering algorithm and wavelet classifier for biometric voice classification," a collection of computational algorithms are proposed to support voice classification; the algorithms are a combination of hierarchical clustering, dynamic time wrap transform, discrete wavelet transform, and decision tree.

In "Using medical history embedded in biometrics medical card for user identity authentication: a privacy preserving authentication model by features matching," authors advocated a novel type of user authentication by using a user's medical history which can be electronically stored in a biometric security card.

In the paper "FGF receptor-mediated gene delivery using ligands coupled to PEI- $\beta-C y D$," authors' current research indicated that the synthesized nonviral vector shows improved gene delivery efficiency and targeting specificity in FGFR-positive cells, indicating that it may have potential applications in cancer gene therapy.

In the paper, "Using medical history embedded in biometrics medical card for user identity authentication: data representation by AVT hierarchical data tree," a new method was proposed for abstracting the medical data by using attribute value taxonomies, into a hierarchical data tree (hData).

In comparison of two "Suspension arrays for simultaneous detection of five biothreat bacterial in powder samples," 
authors reported a suspension array based on the $16 \mathrm{~S}$ rDNA gene amplified by universal primers, which is also called universal primer PCR-based array.

In the paper, "antilisterial activity of nisin-like bacteriocinproducing Lactococcus lactis subsp. lactis isolated from traditional Sardinian dairy products" authors reported the isolation of bacteriocin-like inhibitory substances from LAB strains isolated from artisanal Sardinian dairy products.

Tai-hoon Kim Sabah Mohammed Carlos Ramos Wai-Chi Fang Osvaldo Gervasi Adrian Stoica 

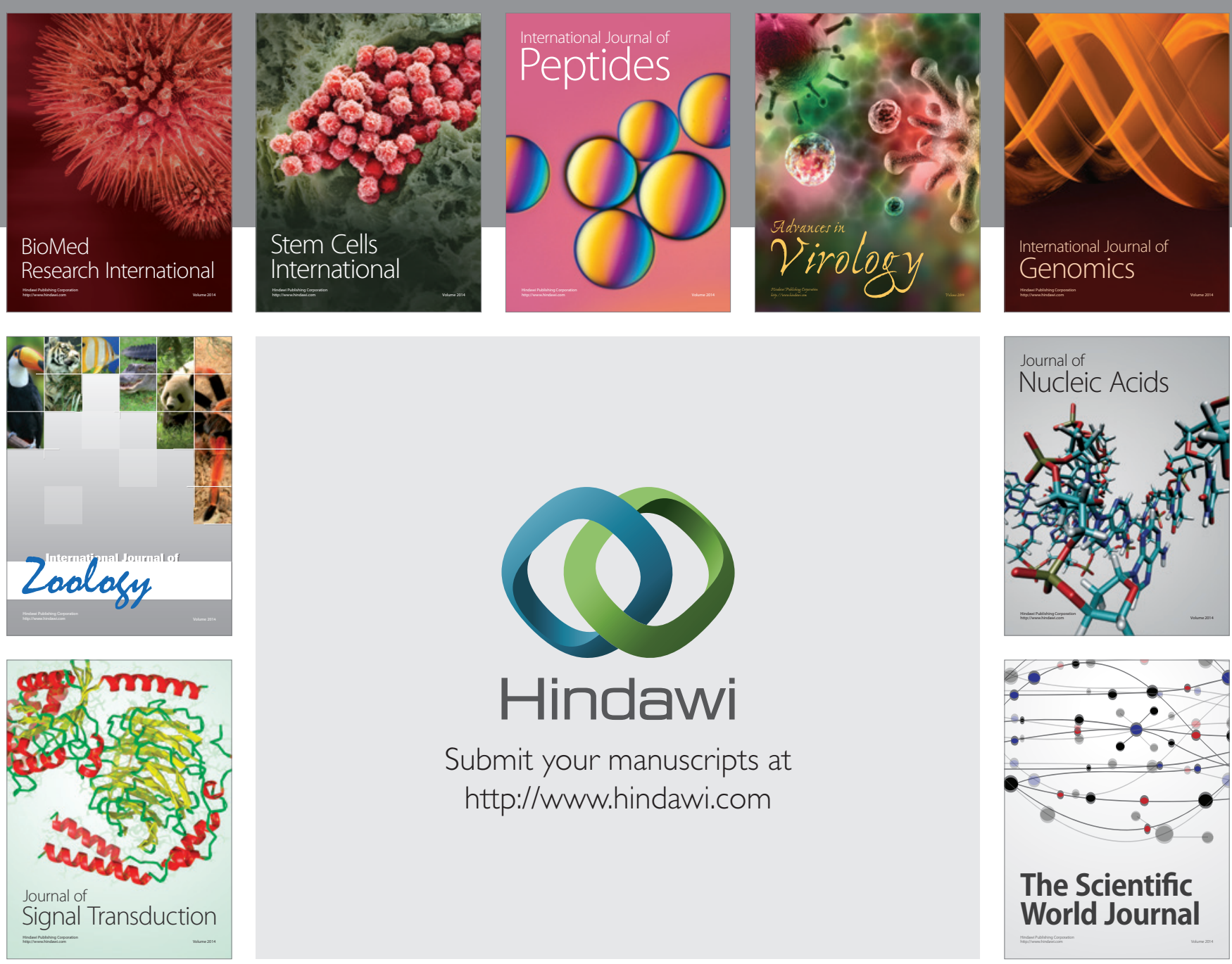

Submit your manuscripts at

http://www.hindawi.com
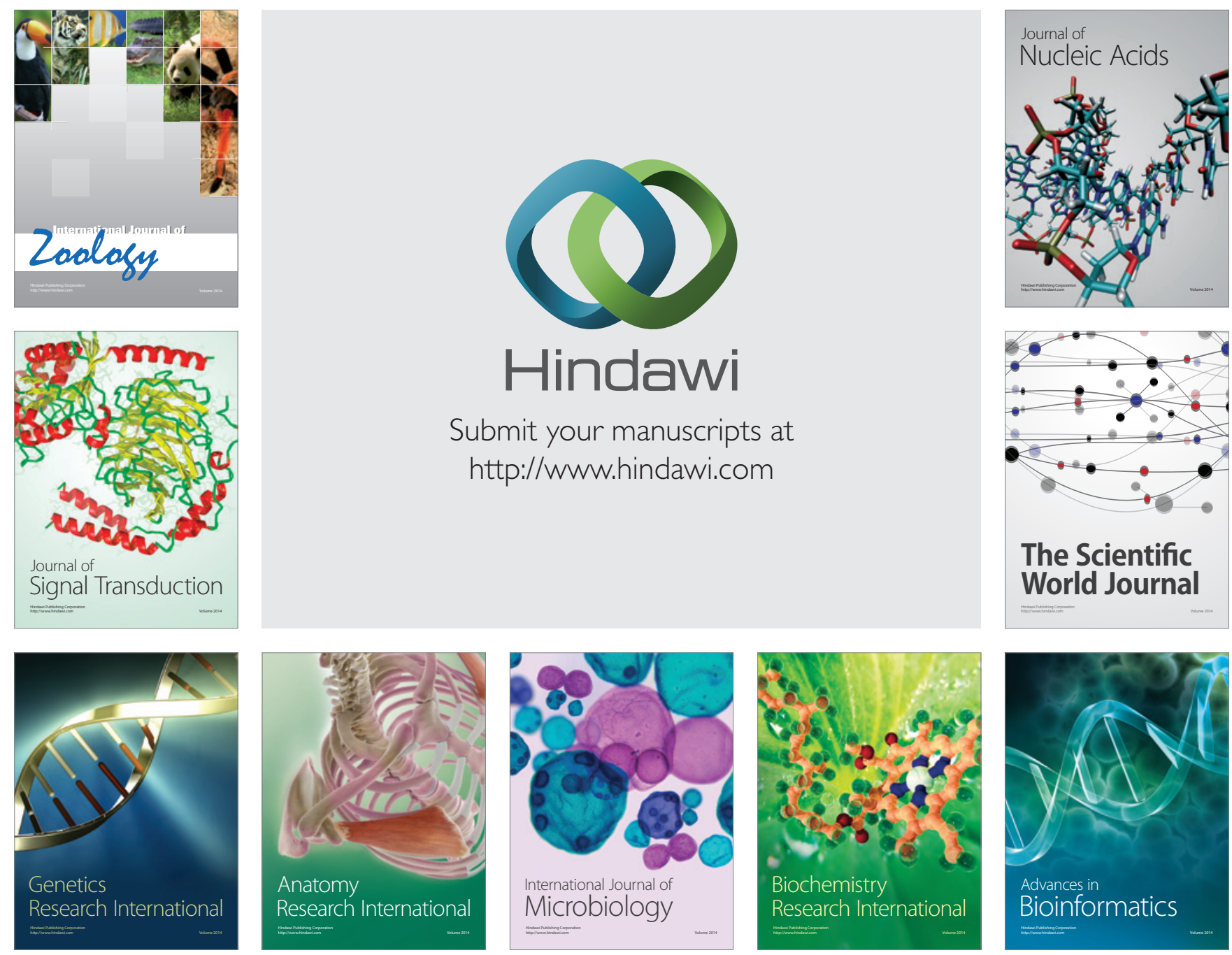

The Scientific World Journal
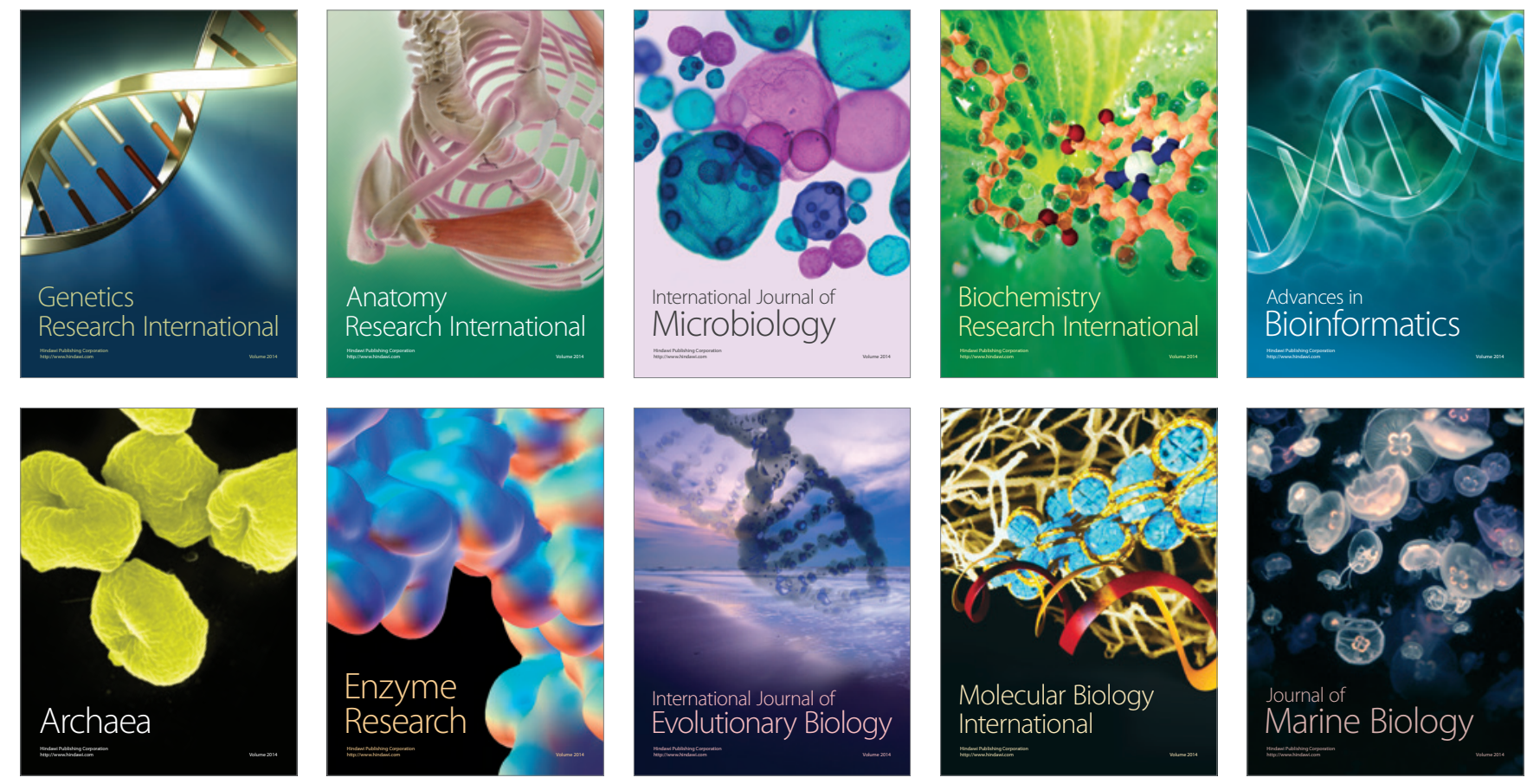